\title{
CERN Calibration, testing, commissioning and first data of ALFA at LHC
}

Sune Jakobsen (Jakobsen@NBI.dk) for the Absolute Luminosity For ATLAS community CERN, PH-ADO, NIELS BOHR INSTITUTE, UNIVERSITY OF COPENHAGEN

\section{Luminosity determination from elastic scattering}

LHC

$<$

$240 \mathrm{~m}$

Aim: Measure the absolute luminosity independent from beam parameters.

Method: Measuring elastic scattering in the Coulomb region (very small angles $\sim 3.5 \mu \mathrm{m}$ ).

Need: Edgeless tracking detector very close to the bream at large distance from the interaction point.

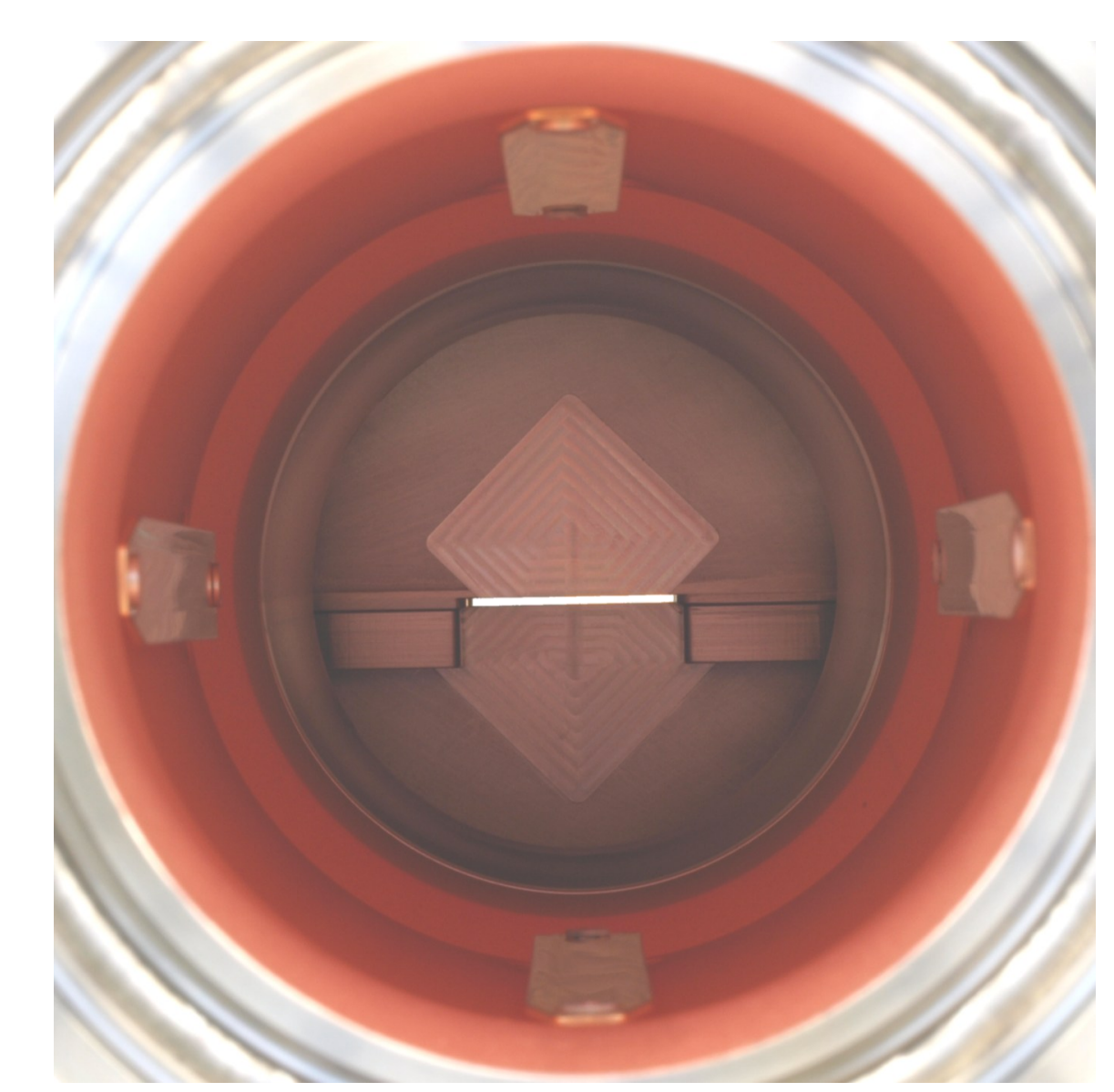

Roman Pots: Metallic vessel separating the detector from the ultra-high beam vacuum.

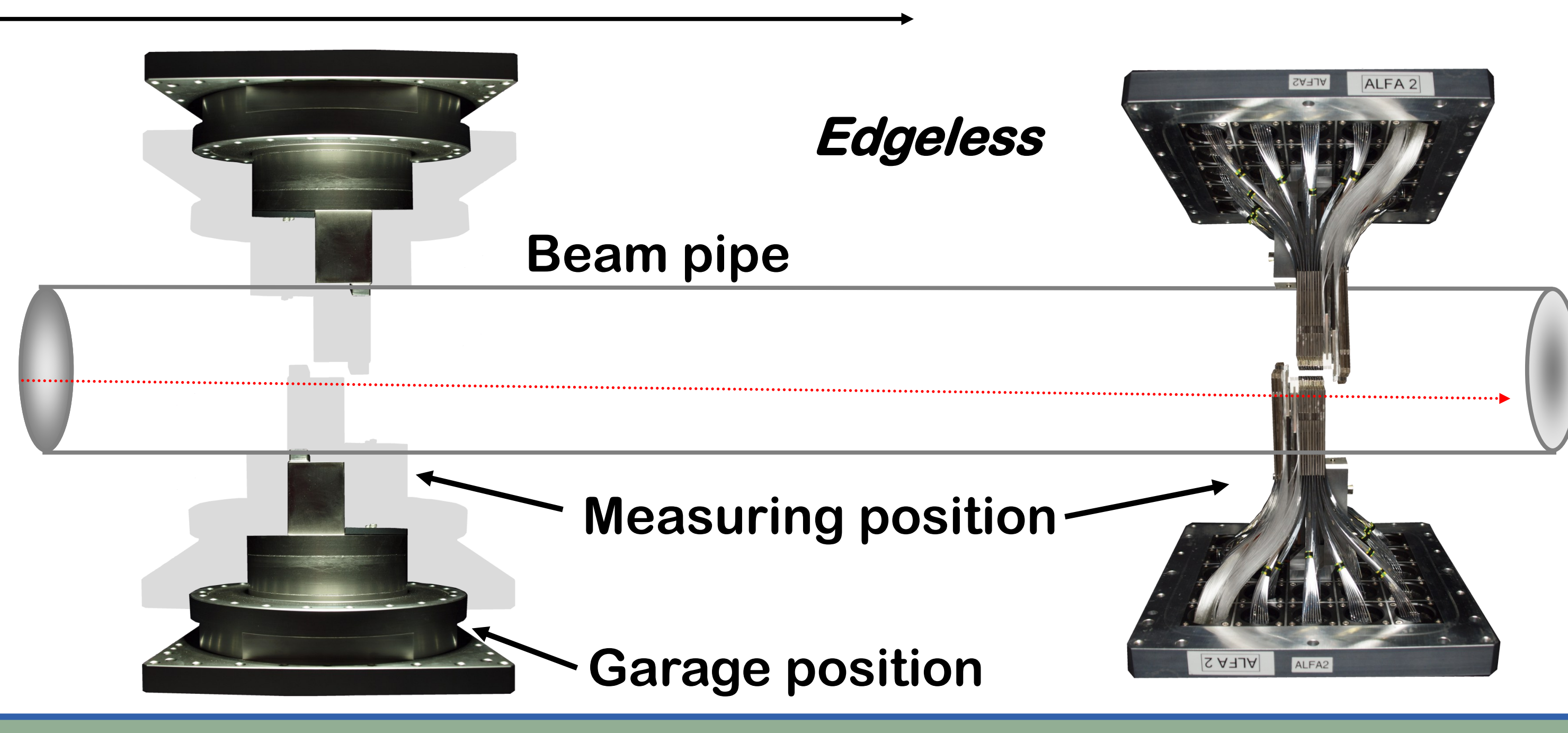

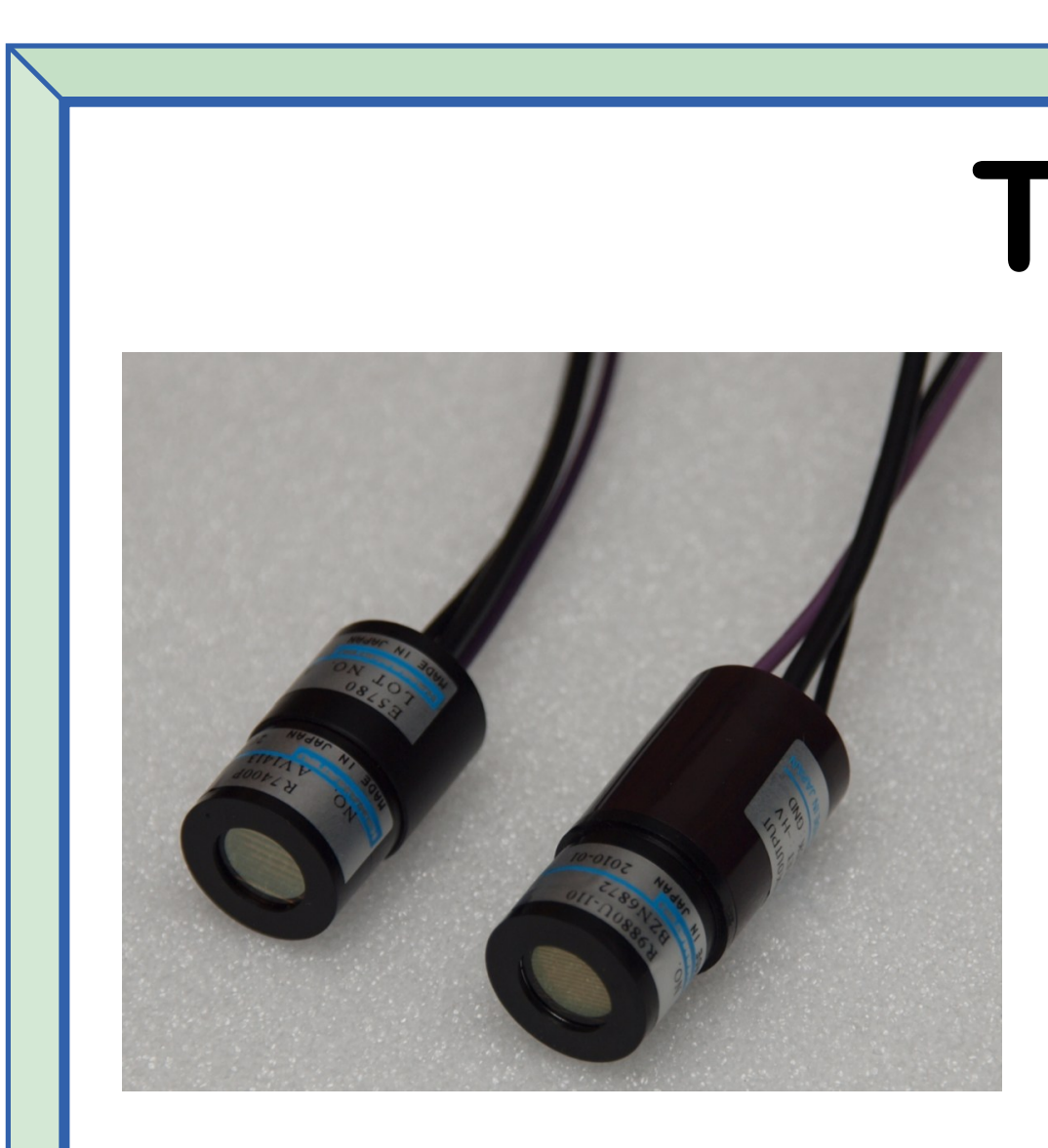

Triggering: Dedicated $3 \mathrm{~mm}$ scintillating tiles readout out by photomultipliers (Hamamatsu R7400P and R9880U-110) are used for triggering.

\section{The ALFA detector system}

Edgeless tracking detector: Scintillating fibers has been chosen which are fully active already at $\sim 20 \mu \mathrm{m}$ from the cut edge. The fiber are positioned in a U-V configuration with 64 fibers in each layer.

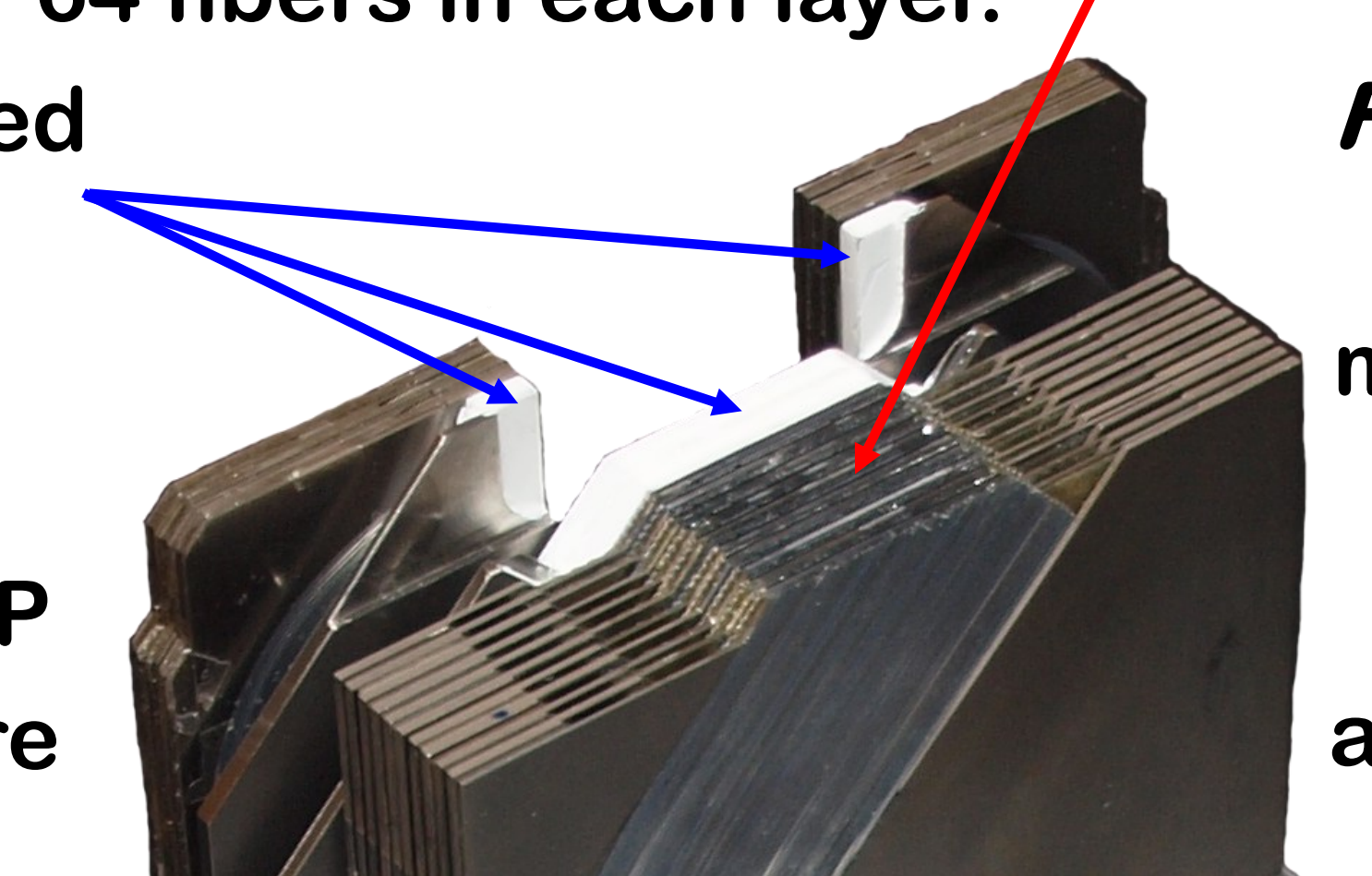

Fiber staggering: To improve detector resolution the aluminized squared scintillating fibers (Kuraray SCSF-78, S-type, $0.5 \times 0.5 \mathrm{~mm}^{2}$ ) are arranged in staggered layers given an overall detector resolution of $\sim 30 \mu \mathrm{m}$. There are a total of - 1460 fibers in each of the 8 detectors.
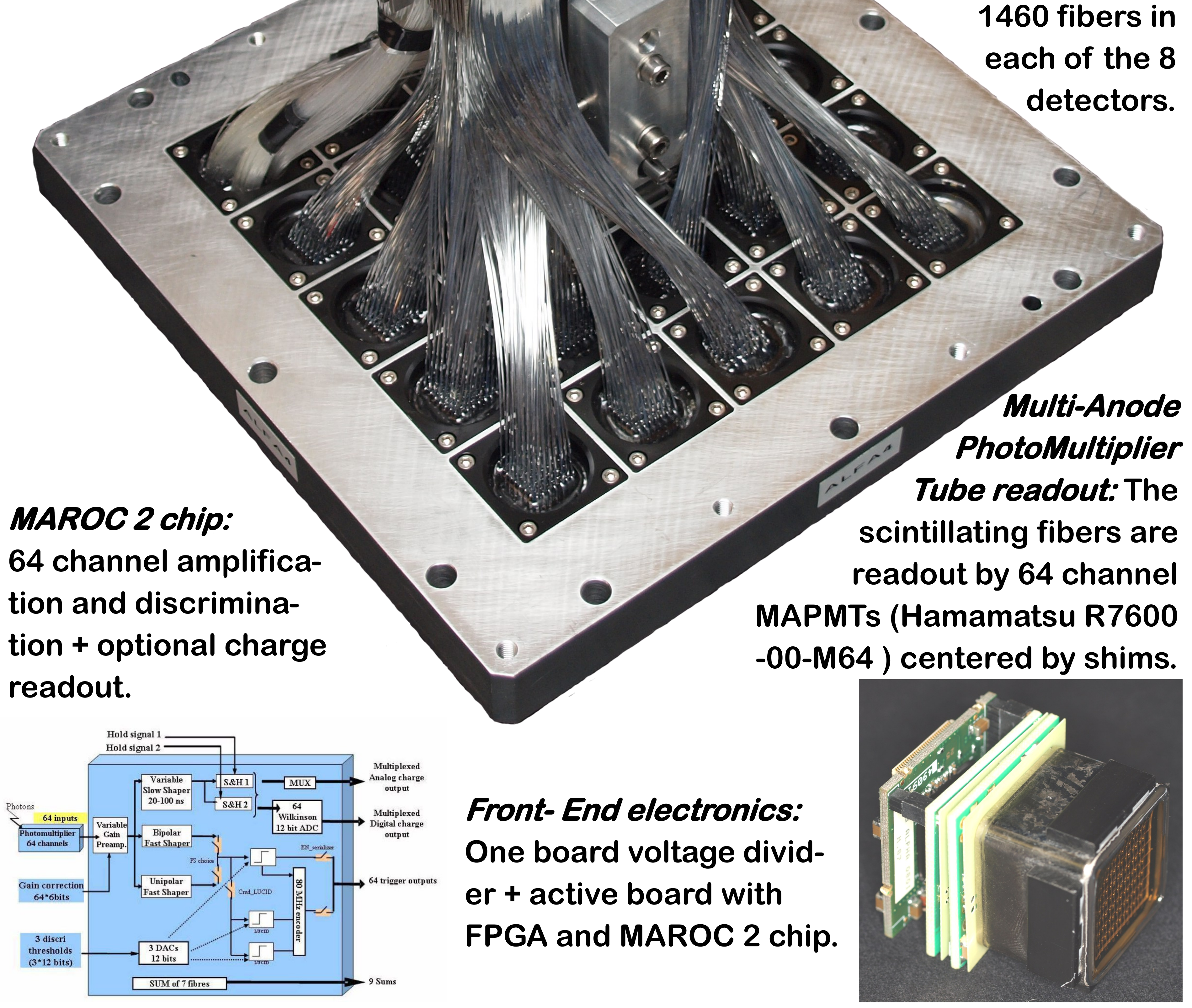

Front- End electronics: One board voltage divider + active board with FPGA and MAROC 2 chip.

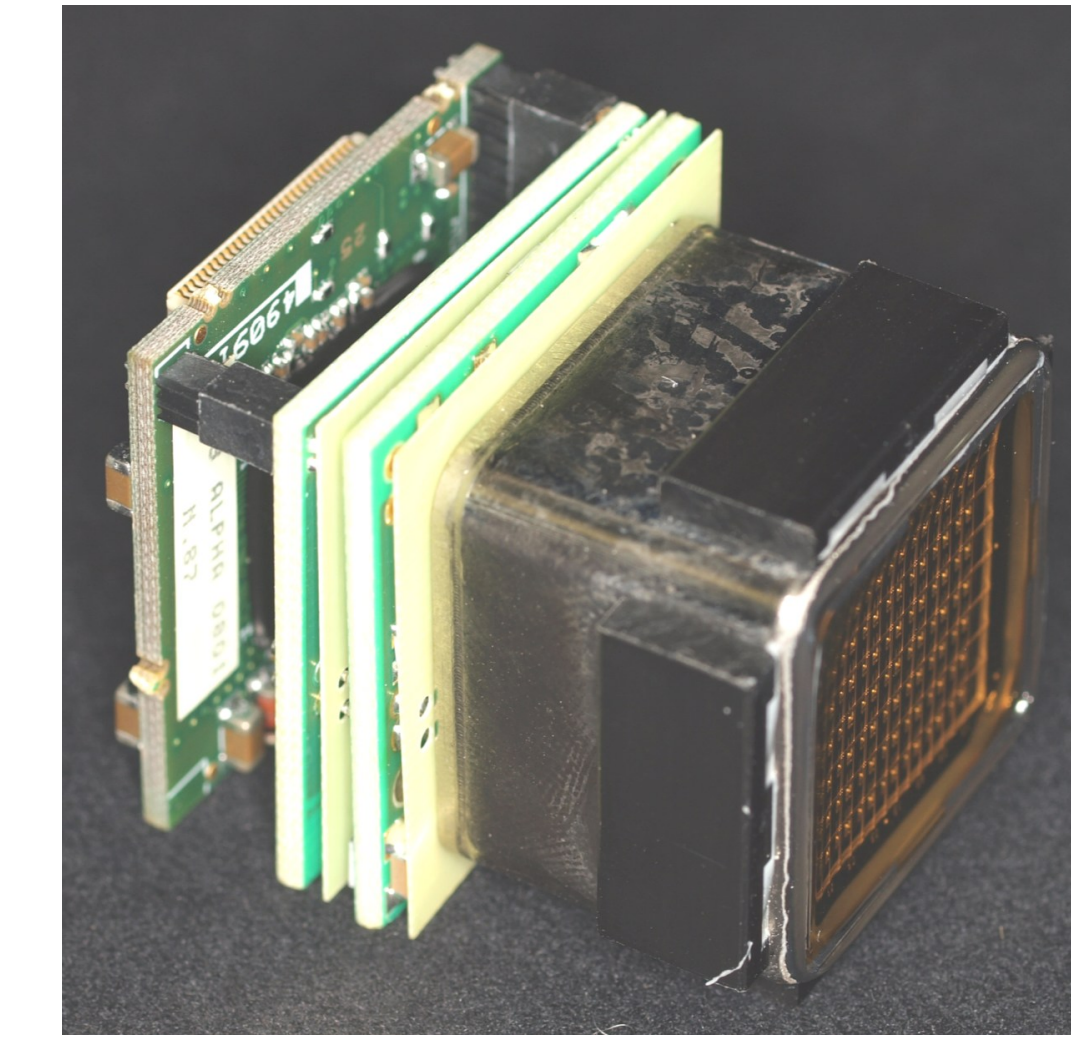

Fiber crosstalk - blackening of Roman Pot window

Test beam data showed much higher than expected fiber crosstalk. Laboratory tests could traced the additional fiber crosstalk back to reflections on the Roman Pot window. Before installation in LHC the Roman Pot window was therefore blackened with $\sim 30 \mu \mathrm{m}$ DAG (graphite) layer.
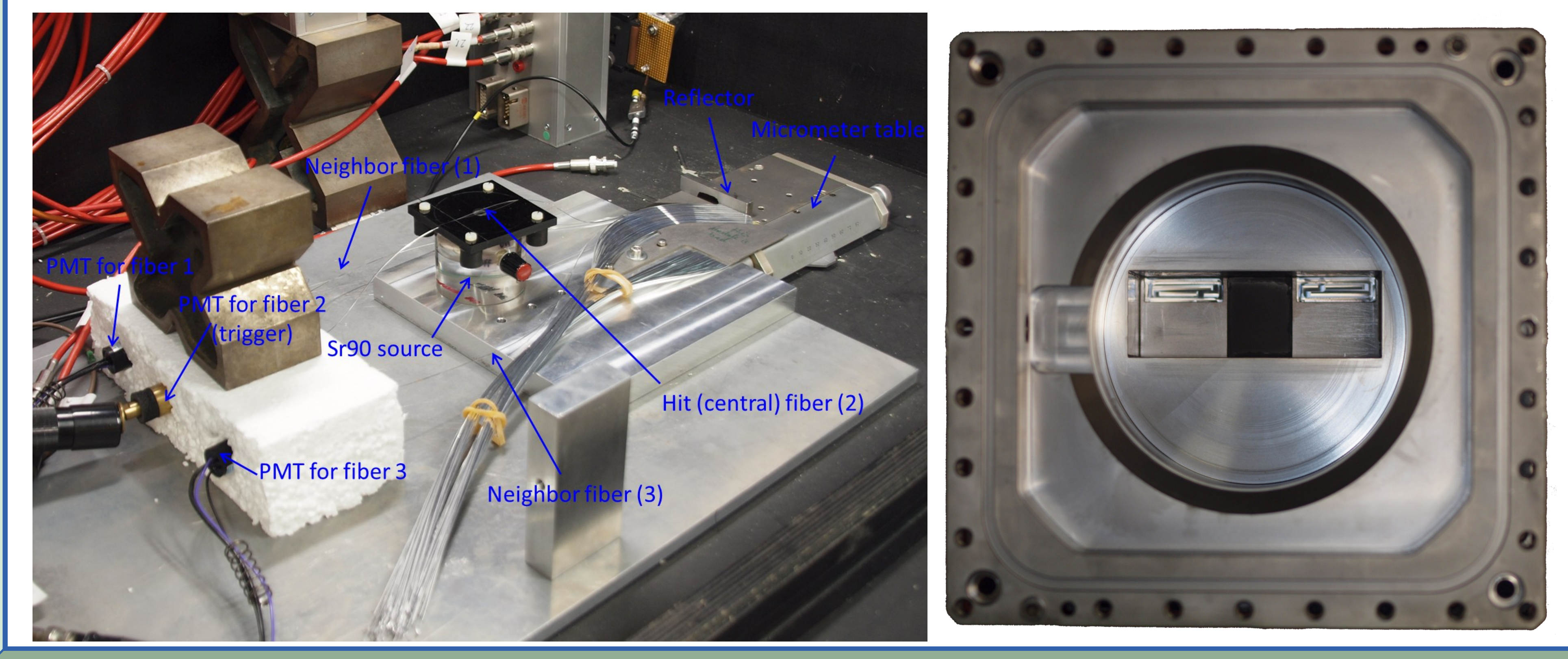

\section{Gain equalization}

The gain of MAPMT channels differs up to a factor 3 and in addition the gain varies about a factor 2 from one MAPMT to another. The position of the

1 photoelectron (PE) is therefore measured using low intensity pulsed LED light. Amplification in the MAROC 2 chip was applied to equalize all channels.

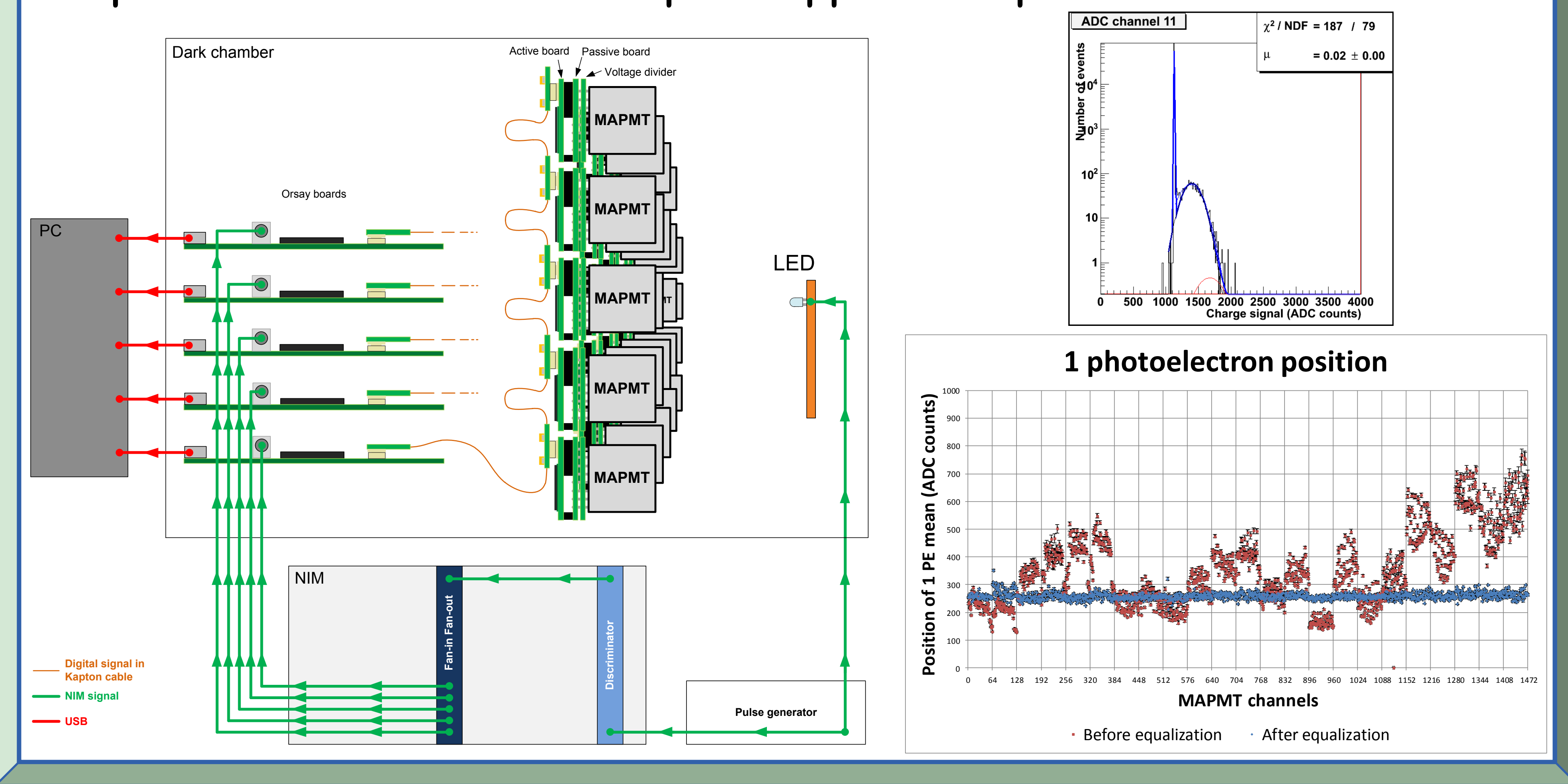

\section{Test with cosmic particles}

The detectors with full Front-End electronics was tested with cosmic particles before installation and the light yield of each scintillating fiber determined.

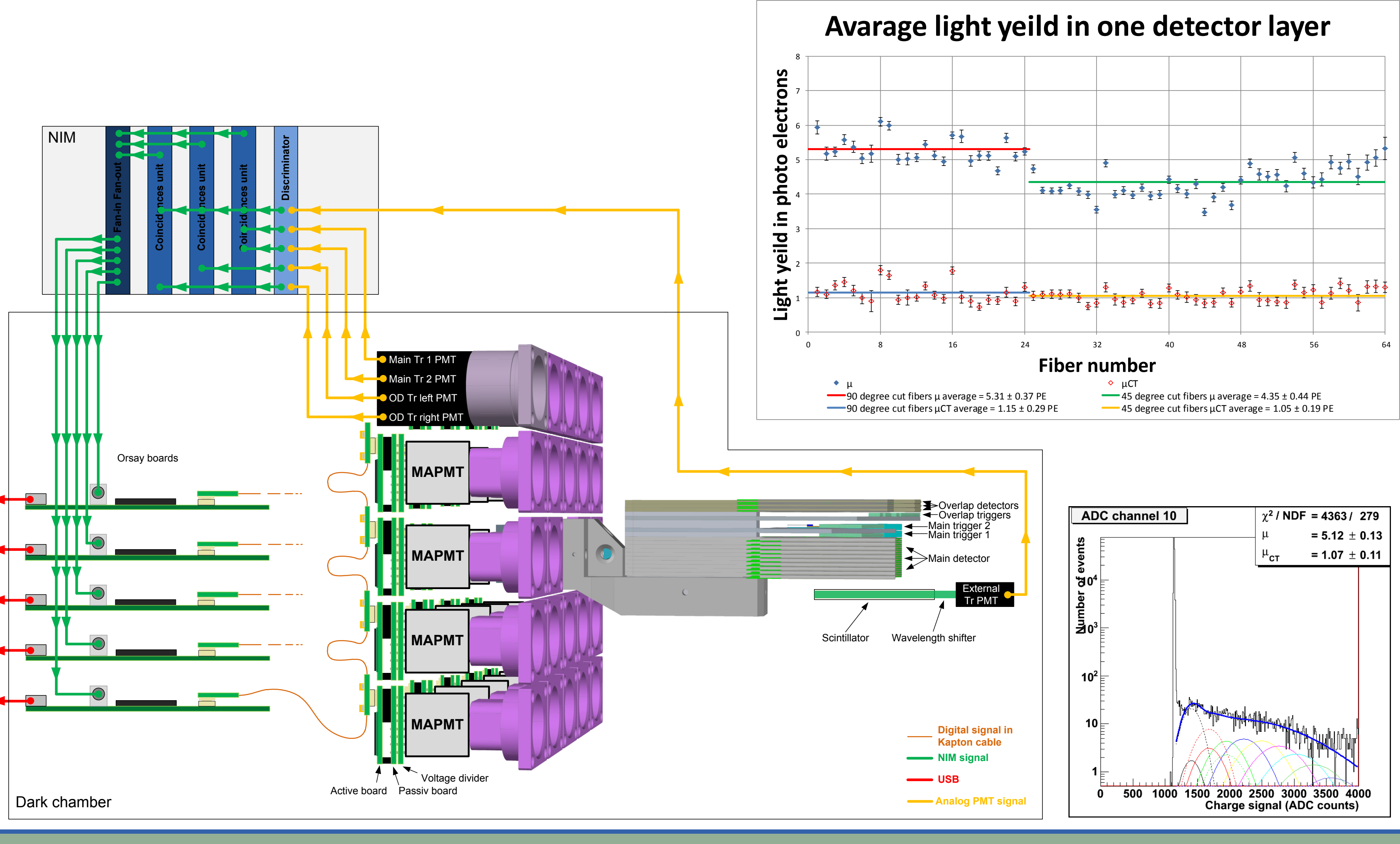

\section{First data from LHC}

All detectors have been installed in the LHC tunnel left and right of ATLAS and the very first data for commissioning have been taking. Left is shown a track in a detector (chosen as it has indication MAPMT crosstalk, which is expected at low level) and right a track map taking with the detector in garage position.
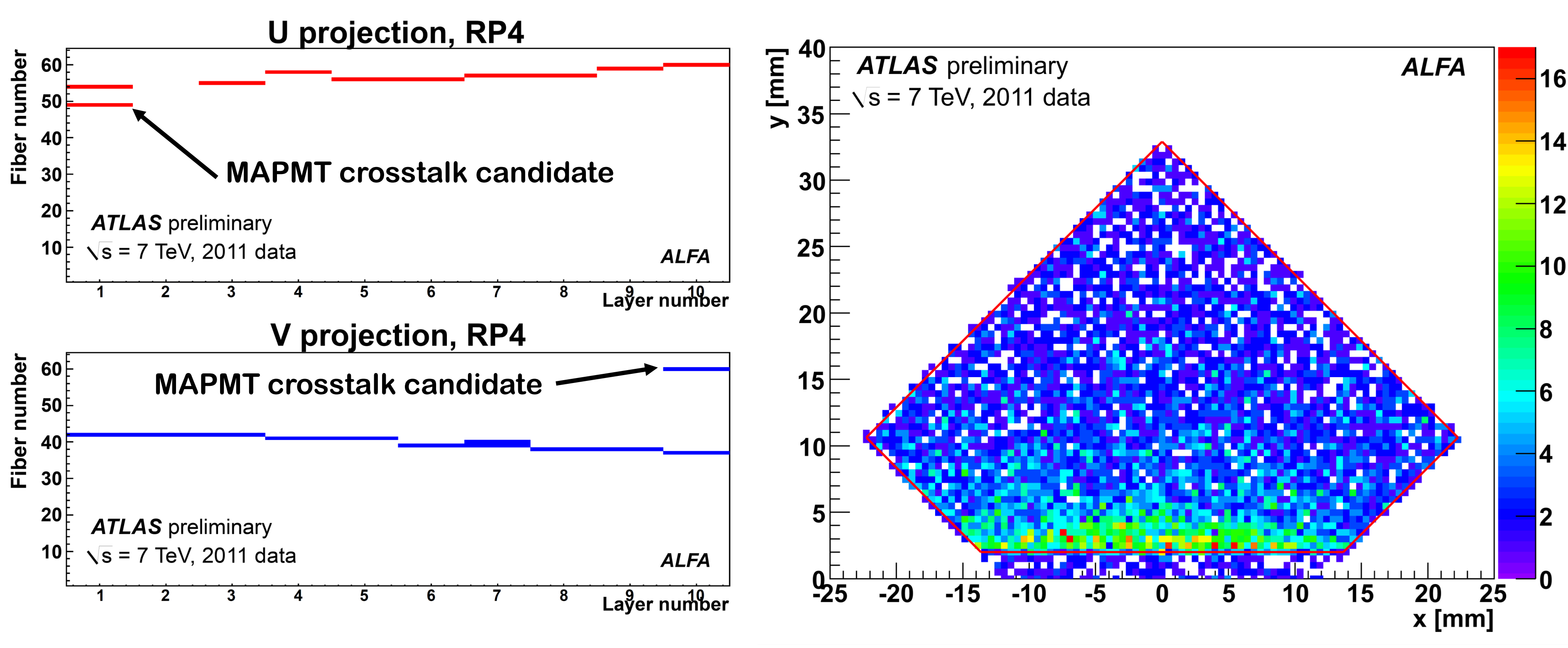